\title{
Oriented Bounding Surfaces with at most Six Common Normals
}

\author{
Margot Rabl, Laureano Gonzalez-Vega, Bert Jüttler and Hans-Peter Schröcker
}

\begin{abstract}
We present a new type of oriented bounding surfaces, which is particularly well suited for shortest distance computations. The bounding surfaces are obtained by considering surfaces whose support functions are restrictions of quadratic polynomials to the unit sphere. We show that the common normals of two surfaces of this type - and hence their shortest distance - can be computed by solving a polynomial of degree six. This compares favorably with other existing bounding surfaces, such as quadric surfaces, where the computation of the common normals is known to lead to a polynomial of degree 24 .
\end{abstract}

\section{INTRODUCTION}

The computation of the minimum distance between two objects is a fundamental task in various applications. These applications include collision detection in robotics [16], [25], interference avoidance (Digital mock-up) in CAD/CAM, interactions in virtual reality and computer games [3], and even interference analysis of molecules in computational physics and computational chemistry [14]. The problem becomes even more challenging if one of the two objects is subject to a motion. In order to obtain realistic simulations, efficient techniques for shortest distance computations are required.

Since it is computationally complicated to apply shortest distance computation directly to general objects, it has become a standard technique to use bounding volumes (or, more generally, hierarchies of bounding volumes) instead. By applying the shortest distance computation to these bounding volumes first, the computational costs can greatly be reduced.

There exist some articles handling curved objects, but they are restricted to some special kinds of surfaces since the minimum distance computation involves a system of non-linear equations. Instead, the majority of the existing literature dealing with minimum distance computation is dedicated to the distance between two polyhedral objects. If the objects of interest have curved boundaries, then they are often approximated by polyhedra.

This work was supported by the Austrian Exchange Service (ÖAD) through the WTZ program ES 111/2007 and the Spanish Program "Acciones Integradas" HU2006-0023 (first three authors). The first and third author were also supported by the Austrian National Research Network on Industrial Geometry (S09202). The second author was also partially supported by the spanish grant MTM2008-04699-C03-03.

M. Rabl and B. Jüttler are with the Institute of Applied Geometry, Johannes Kepler University, A-4040 Linz, Austria \{margot.rabl, bert. juettler\}ajku. at

L. Gonzalez-Vega is with the Department of Mathematics, Statistics and Computing, Universidad de Cantabria, ES-39005 Santander, Spain laureano.gonzalez@unican.es

H.P. Schröcker is with the Institute of Basic Sciences in Engineering, Unit Geometry and CAD, University Innsbruck, A-6020 Innsbruck, Austria hans-peter.schroeckereuibk.ac.at
The simplest bounding polyhedra are axis-aligned bounding boxes (AABBs), which are easy to construct for a given object and provide a fast and simple intersection test. On the other hand, the approximation quality is relatively low and the recomputation for moving objects is required.

The use of oriented bounding boxes (OBBs) avoids the recomputation and provides better approximation quality. However, the data volume needed for representing OBBs is larger than for AABBs and the intersection test is relatively complicated. See [4] for more information.

Klosowski et al. [9] proposed to use collections of discrete orientation polytopes (DOPs) as bounding volumes. But generally speaking, one needs a large data volume, which may entail problems with the efficiency, in order to obtain precise results. For avoiding this blow-up of data volume, it has been proposed to use bounding ellipsoids and piecewise quadric objects [22], as well as other geometric primitives.

Using enclosing ellipsoids, the problems of collision avoidance and detection are studied in [7], [18], [24]. Sohn et al. [21] formulate the task of distance computation as a surface-surface intersection problem in the space of lines. Lennerz and Schömer [10] provide a method for distance computation of generalized polyhedra bounded by patches of quadratic surfaces trimmed by quadratic curves. Their main result states that the distance between two faces of quadratic complexes can be computed by solving a univariate polynomial equation of degree at most 24 , which is found using a Lagrangian mutliplier technique.

Seong et al. [20] compute the distance between two simple surfaces i.e. surfaces of revolution and surfaces of linear extrusion, generated by slope-monotone curves. The main advantage of these simple surfaces is the simplicity of their Gauss maps, which makes the normal matching relatively easy to determine.

Chen et al. [1] compute the distance of two implicit algebraic surfaces by using an offsetting technique. They reduce the problem of distance computation between a quadric surface in implicit representation and (a) a cylinder, (b) a cone, (c) an elliptic paraboloid, (d) an ellipsoid and (e) a torus to the problem of solving a univariate polynomial of degree (a) 4, (b) 8, (c) 16, (d) 36 and (e) 16. This paper presents a detailed comparison of the methods from [8], [10], [21] and of the offsetting-based method.

Lee et al. [11] replace the problem of finding the minimum distance between two sphere-swept surfaces by computing the distance between two moving spheres. The resulting nonlinear system of multivariate rational functions is solved iteratively by a special subdivision method.

Odehnal [13] provided a solution for finding all common 
normals of two tori via line geometry. In general two different tori possess 8 common normals, but in some special cases, they can have infinitely many. Detailed analysis of these special tori positions is presented.

Choi et al. [2] solved the problem of collision detection of two moving ellipsoids under rational motions. Their method is based on an algebraic condition that arises from the characteristic polynomial of two ellipsoids, which is a polynomial of degree 4. By just determining the existence of negative roots, without computing them explicitly, it is possible to determine, whether the ellipsoids are separate or not. If the ellipsoids collide during the rational motion, then they can report the collision intervals by computing zero-sets as well.

In this paper we propose a new type of oriented bounding surface for distance-distance computation. It is a special surface with polynomial support functions, which were analyzed in more detail in [19]. Support functions are a special concept from convex geometry [6], and their application to problems of geometric computing can be traced back to a classical paper of Sabin [17], see also [5]. We restrict our attention to surfaces with a polynomial support function of degree two, which are called quadratically supported surfaces (QSS). Using Hermite interpolation with spherical PowellSabin elements, segments of such surfaces are capable of approximating general support functions [19].

The class of surfaces is closed under important geometric operations (translation, rotation, offsetting) and it includes both convex and non-convex objects. In particular it provides an interesting type of non-convex surface of revolution, which may provide a tight bound of manipulator-type objects.

In a recent paper we demonstrated that these surfaces are potentially interesting for kinematics and robotics, since the characteristic curves forming the envelope of a moving QSS can be computed exactly [12]. In the present paper we explore the potential of QSS for shortest distance computation.

Figure 1 introduces an example, to be treated in more detail in Section VI, which demonstrates the potential of the new type of bounding volumes. The manipulator has been modeled as a collection of two non-convex QSS of revolution and three spheres and the shortest distance problem has been solved by determining the common normals between these QSS.
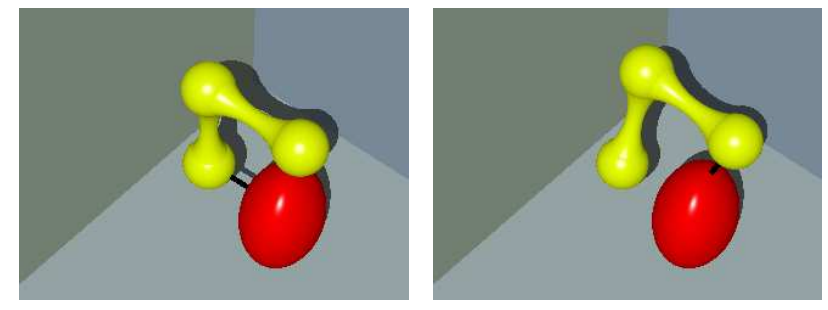

Fig. 1. Shortest distance (black line segment) between a moving manipulator (yellow) and an obstacle (red), both represented by QSS.

The remainder of this paper is organized as follows. First we recall the support function representation of surfaces and analyze the shapes which can be obtained from the class of QSS. Then we address the problem of shortest distance computation and show that all common normals can be found by solving a univariate polynomial equation of degree 6 . Finally we provide some examples, additional information about the planar case and we conclude the paper.

\section{SUPPORT FunCTIONS}

We recall the support function representation of surfaces, which is a classic concept from the field of convex geometry (see e.g. [19]). Consider a given function $h \in C^{\infty}\left(\mathbb{S}^{2}, \mathbb{R}\right)$, where $\mathbb{S}^{2}$ denotes the unit sphere in $\mathbb{R}^{3}$. We use this function to associate with each point $\mathbf{n} \in \mathbb{S}^{2}$ the plane

$$
\left\{\mathbf{p} \in \mathbb{R}^{3}: \mathbf{p} \cdot \mathbf{n}=h(\mathbf{n})\right\}
$$

which has the unit normal $\mathbf{n}$ and possesses the oriented distance $h(\mathbf{n})$ to the origin.

A surface can now be defined as the envelope of the twoparameter family of planes, which is obtained by varying $\mathbf{n}$ in $\mathbb{S}^{2}$. Along with the surface we get an oriented unit normal vector at each point, hence we get an oriented surface.

The given function $h$ is called the support function of this surface. For any $h \in C^{\infty}\left(\mathbb{S}^{2}, \mathbb{R}\right)$, a parameterization $\mathbf{x}_{h} \in C^{\infty}\left(\mathbb{S}^{2}, \mathbb{R}\right)$ of the surface is given by its inverse Gauss map,

$$
\mathbf{x}_{h}(\mathbf{n})=h(\mathbf{n}) \mathbf{n}+\left(\nabla_{\mathbb{S}^{2}} h\right)(\mathbf{n})
$$

where $\left(\nabla_{\mathbb{S}^{2}} h\right)$ is the intrinsic gradient of the support function $h$ with respect to the unit sphere $\mathbb{S}^{2}$. If the support function $h$ is obtained by restricting a suitable function $h^{0} \in C^{\infty}\left(\mathbb{R}^{3}, \mathbb{R}\right)$ to the unit sphere $\mathbb{S}^{2}$, then

$$
\left(\nabla_{\mathbb{S}^{2}} h\right)(\mathbf{n})=\left(\nabla h^{0}\right)(\mathbf{n})-\left[\left(\nabla h^{0}\right)(\mathbf{n}) \cdot \mathbf{n}\right] \mathbf{n},
$$

where $\nabla$ is the usual nabla operator in $\mathbb{R}^{3}$. This parameterization, whose domain is the unit sphere, can now be composed with any parameterization of $\mathbb{S}^{2}$, e.g., with spherical coordinates. This gives a representation of the surface $\mathbf{x}_{h}$ as a parametric surface.

In this paper we are particularly interested in the case where the support function is the restriction of a trivariate quadratic polynomial to $\mathbb{S}^{2}$,

$$
\begin{aligned}
h(\mathbf{n})= & a_{1} x^{2}+a_{2} y^{2}+a_{3} z^{2}+a_{4} x y+a_{5} x z+a_{6} y z+ \\
& a_{7} x+a_{8} y+a_{9} z+a_{10}
\end{aligned}
$$

where $\mathbf{n}=(x, y, z)$. We call the corresponding envelopes quadratically supported surfaces (QSS).

A translation of the envelope surface by a vector $\mathbf{v}$ corresponds to the addition of the homogeneous linear polynomial $\mathbf{v} \cdot \mathbf{n}$ to the support function, while a rotation can immediately be composed with it. Moreover, addition of constants corresponds to offsetting. Finally, the QSS with the opposite orientation has the support function

$$
h^{*}(\mathbf{n})=-h(-\mathbf{n}) .
$$

We note that the class of QSS is closed under translations, rotations, offsetting and orientation reversal. 
A QSS depends on 9 free parameters, hence it has the same number of degrees of freedom as a general quadric surface. Indeed, the support function in Eq. (4) has 10 coefficients, but the coefficient of the constant term can be multiplied by $\mathbf{n}^{2}=x^{2}+y^{2}+z^{2}$, which gives 1 when restricted to the unit sphere. Consequently, only the coefficients of the linear and of the quadratic terms represent independent degrees of freedom.

\section{THE SHAPES OF QSS}

We analyze the possible shapes which can be obtained from quadratic QSS. First we observe that any quadratic support function has the normal form

$$
h(\mathbf{n})=x^{2}+a y^{2}+b z^{2}
$$

with constant coefficients $a, b \in \mathbb{R}$. Indeed, the linear terms of $h$ can be eliminated by a translation, the constant term can be added to the quadratic ones by multiplying it with $1=x^{2}+y^{2}+z^{2}$ and the quadratic terms can be diagonalized by a rotation. Finally, a scaling can be applied to normalize the coefficient of $x^{2}$.

Consequently, the shapes of the QSS depend on two coefficients $a$ and $b$. In order to classify these shapes, we analyze the set of singular points on a QSS. We compose a quadratic rational parameterization of the unit sphere with the inverse Gauss map. This gives the parameterization

$$
\mathbf{q}_{h}(u, v)=\mathbf{x}_{h}\left(\frac{1-u^{2}-v^{2}}{1+u^{2}+v^{2}}, \frac{2 u}{1+u^{2}+v^{2}}, \frac{2 v}{1+u^{2}+v^{2}}\right)
$$

of the QSS which is defined by the support function (6). The parameters $(u, v)$ vary in $\mathbb{R}^{2}$.

The singular points of this parameterization are characterized by

$$
f_{h}(u, v)=\left\|\frac{\partial}{\partial u} \mathbf{q}_{h} \times \frac{\partial}{\partial v} \mathbf{q}_{h}\right\|^{2}=0 .
$$

This equation defines an algebraic curve of degree 8 in the $u v$ plane, whose coefficients depend polynomially on $a$ and $b$. We will call this curve the singularity curve $\mathcal{S}$ of the QSS given by the parameterization $\mathbf{q}_{h}$.

The study of the different shapes that can be obtained from a quadratic QSS is guided by the analysis of the singularity curve. Figure 2 shows the singularity curve $\mathcal{S}$ together with the corresponding QSS when $a=2$ and $b=5$.
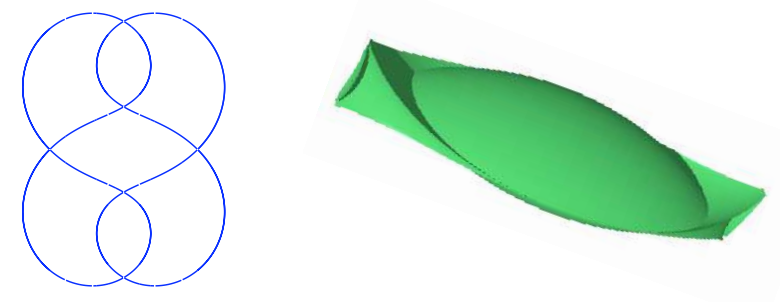

Fig. 2. Singularity curve (left) and QSS (right) when $a=2$ and $b=5$.

Next we include several relevant cases showing how the study of the singularity curve $\mathcal{S}$ produces the desired shapes.
We refrain from presenting a complete classification of all available shapes, since this is beyond the scope of the present paper.

A. Convex and non-singular QSS: $(a, b) \in\left(\frac{1}{2}, 2\right) \times\left(\frac{1}{2}, 2\right)$ such that $a \leq 2 b \wedge b \leq 2 a$

In this case the curve $f_{h}(u, v)=0$ is empty and the corresponding QSS is free of singularities and convex (see Figure 3). In particular, we obtain a sphere if $a=b=1$.

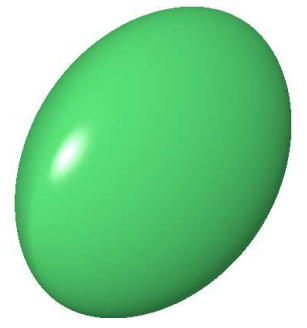

Fig. 3. Shape of the convex and non-singular QSS when $a=31 / 16$ and $b=3 / 2$.

B. QSS which are surfaces of revolution: $a=b=k \in \mathbb{R}$, $a=1 \wedge b \in \mathbb{R}$ and $b=1 \wedge a \in \mathbb{R}$

Let us consider the case $a=b=k \in \mathbb{R}$, where the corresponding QSS are surfaces of revolution. The singularity curve $f_{h}(u, v)$ factors

$$
\begin{aligned}
& \left((2 k-1)\left(u^{2}+v^{2}\right)^{2}+2\left(u^{2}+v^{2}\right)+(2 k-1)\right) \\
& \cdot\left((2 k-1)\left(u^{2}+v^{2}\right)^{2}+2(5-4 k)\left(u^{2}+v^{2}\right)+(2 k-1)\right)
\end{aligned}
$$

providing seven possible configurations for the singularity curve (circles and points). The different classes of shapes of the QSS when $a=b=k \in \mathbb{R}$ can be seen in Figure 4 . The two other symmetric cases give similar shapes of QSS.

\section{COMMON NORMALS AND SHORTEST DISTANCES}

We consider two quadratic support functions

$$
\begin{aligned}
g(\mathbf{n})= & a_{1} x^{2}+a_{2} y^{2}+a_{3} z^{2}+a_{4} x y+a_{5} x z+a_{6} y z+ \\
& a_{7} x+a_{8} y+a_{9} z+a_{10} \text { and } \\
h(\mathbf{n})= & b_{1} x^{2}+b_{2} y^{2}+b_{3} z^{2}+b_{4} x y+b_{5} x z+b_{6} y z+ \\
& b_{7} x+b_{8} y+b_{9} z+b_{10},
\end{aligned}
$$

with constant coefficients $a_{i}, b_{i} \in \mathbb{R}$ for $i=1 \ldots 10$. They define two QSS

$$
\begin{aligned}
& \mathbf{x}_{g}(\mathbf{n})=g(\mathbf{n}) \mathbf{n}+\nabla g(\mathbf{n})-[\nabla g(\mathbf{n}) \cdot \mathbf{n}] \mathbf{n} \text { and } \\
& \mathbf{x}_{h}(\mathbf{n})=h(\mathbf{n}) \mathbf{n}+\nabla h(\mathbf{n})-[\nabla h(\mathbf{n}) \cdot \mathbf{n}] \mathbf{n} .
\end{aligned}
$$

Definition 1: Both QSS possess a common normal if the two vectors

$$
\left(\mathbf{x}_{g}(\mathbf{n})-\mathbf{x}_{h}(\mathbf{n})\right) \text { and } \mathbf{n}
$$

are linearly dependent.

Note that the unit normals (both are $\mathbf{n}$ ) at the two surface points $\mathbf{x}_{g}(\mathbf{n})$ and $\mathbf{x}_{h}(\mathbf{n})$ are required to be parallel and to possess the same orientation.

Consequently, if one computes the shortest distance of two convex QSS, then their orientation has to be chosen such that the normal vectors of one surface point inward, while the normal vectors of the other surface point outward. Then 

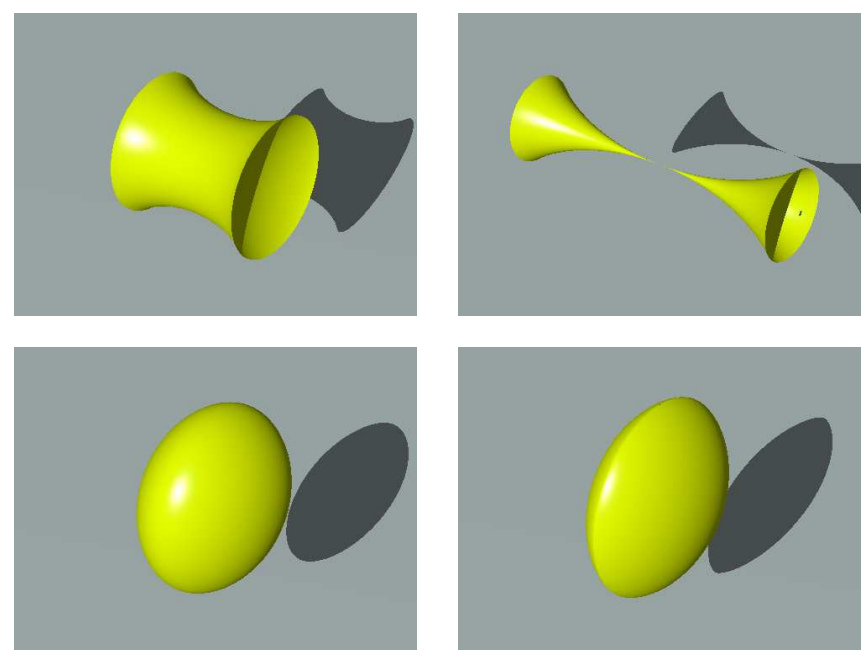

the shortest distance will be realized at one of the common normals in the sense of this definition.

In the case of non-convex QSS, both possible orientations, which are related by (5), have to be analyzed. In addition, the singular curves on the surface have to be dealt with separately (unless they can be excluded by other reasons).

Since the first and third terms in (8) are all linearly dependent on $\mathbf{n}$, the condition of Definition 1 is equivalent to

$$
(\nabla g(\mathbf{n})-\nabla h(\mathbf{n})) \times \mathbf{n}=\mathbf{0} .
$$

In the case of two QSS, this equation gives the system

$$
\begin{aligned}
0= & c_{4}\left(-x^{2}+y^{2}\right)+2\left(c_{1}-c_{2}\right) x y-c_{6} x z+c_{5} y z \\
& -c_{8} x+c_{7} y \\
0= & c_{6}\left(-y^{2}+z^{2}\right)-c_{5} x y+c_{4} x z+2\left(c_{2}-c_{3}\right) y z \\
& -c_{9} y+c_{8} z \\
0= & c_{5}\left(-x^{2}+z^{2}\right)-c_{6} x y+2\left(c_{1}-c_{3}\right) x z+c_{4} y z \\
& -c_{9} x+c_{7} z
\end{aligned}
$$

of three quadratic equations, where $c_{i}=\left(a_{i}-b_{i}\right)$ for $i=$ $1 \ldots 9$. Together with the condition

$$
\mathbf{n} \cdot \mathbf{n}=x^{2}+y^{2}+z^{2}=1
$$

for normalization, we arrive at a system of 4 quadratic equations in 3 unknowns. Due to the special position of the quadrics described by the three equations (11), one may expect to find not more than $2^{3}=8$ solutions $\mathbf{n}_{j}$, $j=1, \ldots, 8$. These solutions are the unit normals of the common normals, which pass through the surface points $\mathbf{x}_{g}\left(\mathbf{n}_{j}\right)$ and $\mathbf{x}_{h}\left(\mathbf{n}_{j}\right)$.

The number of solutions, which is always less than 8 , will be analyzed in the next section.

\section{NUMBER AND COMPUTATION OF COMMON NORMALS}

We denote the three quadratics in Eq. 11 by $Q_{1}, Q_{2}$ and $Q_{3}$, respectively. Recall that two general quadrics intersect in a quartic space curve, see e.g. [23]. In our case, however, we have the following result.
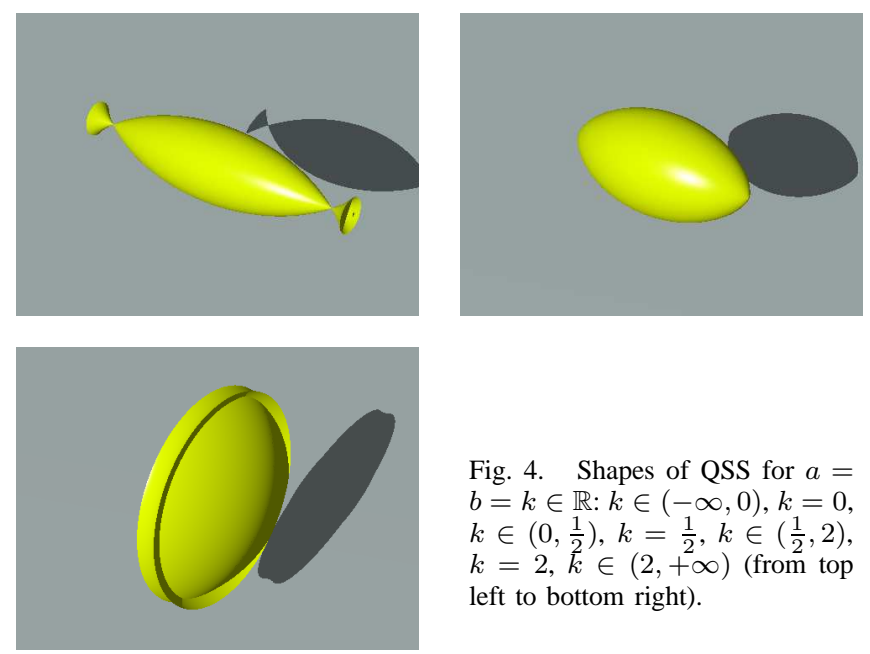

Fig. 4. Shapes of QSS for $a=$ $b=k \in \mathbb{R}: k \in(-\infty, 0), k=0$, $k \in\left(0, \frac{1}{2}\right), k=\frac{1}{2}, k \in\left(\frac{1}{2}, 2\right)$, $k=2, k \in(2,+\infty)$ (from top left to bottom right).

Lemma 1: Any two of the three quadrics $Q_{1}, Q_{2}$ and $Q_{3}$ intersect in a cubic curve and in a line.

Proof: It suffices to show that each of the three intersection curves contains a line. The two quadrics $Q_{1}$ and $Q_{2}$ intersect in the line

$$
y=0 \quad \text { and } \quad c_{4} x+c_{6} z+c_{8}=0,
$$

the two quadrics $Q_{2}$ and $Q_{3}$ intersect in the line

$$
z=0 \quad \text { and } \quad c_{5} x+c_{6} y+c_{9}=0
$$

and the remaining two quadrics $Q_{1}$ and $Q_{3}$ intersect in the line

$$
x=0 \quad \text { and } \quad c_{4} y+c_{5} z+c_{7}=0 .
$$

Consequently, each of the three intersection curves splits into a cubic curve and a line.

As the next step we analyze the three cubic curves and show that they are all identical.

Lemma 2: The three quadrics $Q_{1}, Q_{2}$ and $Q_{3}$ intersect in one cubic curve.

Proof: In order to simplify the notation we shall use homogeneous coordinates $\left(d_{0}, d_{1}, d_{2}, d_{3}\right)^{\top}$ defined by

$$
1: x: y: z=d_{0}: d_{1}: d_{2}: d_{3}
$$

to represent points. Let $l_{1}$ and $l_{2}$ be the line (13) contained in $Q_{1}$ and $Q_{2}$ and the line (14) contained in $Q_{2}$ and $Q_{3}$, respectively. In homogeneous coordinates, two possible parametric representations of these two lines are

$$
\mathbf{p}(s)=\mathbf{p}_{0}+s \mathbf{p}_{1}, s \in \mathbb{R} \quad \text { and } \quad \mathbf{q}(t)=\mathbf{q}_{0}+t \mathbf{q}_{1}, t \in \mathbb{R}
$$

with the points $\mathbf{p}_{0}=\left(-c_{6}, 0,0, c_{8}\right)^{\top}, \mathbf{p}_{1}=\left(-c_{4}, c_{8}, 0,0\right)^{\top}$ and $\mathbf{q}_{0}=\left(-c_{6}, 0, c_{9}, 0\right)^{\top}, \mathbf{q}_{1}=\left(-c_{5}, c_{9}, 0,0\right)^{\top}$. For any point $\mathbf{p}(s)$ on the first line, we can find a unique point $\mathbf{q}(t)$ on the second line such that the line spanned by them

$$
\mathbf{r}(s, t, u)=\mathbf{p}+u \mathbf{q}
$$


is completely contained in the second quadric $Q_{2}$. In homogeneous coordinates, the second quadric has the representation $0=\mathbf{x}^{\top} C_{2} \mathbf{x}$ with the $4 \times 4$-matrix

$$
C_{2}=\frac{1}{2}\left(\begin{array}{cccc}
0 & 0 & -c_{9} & c_{8} \\
0 & 0 & -c_{5} & c_{4} \\
-c_{9} & -c_{5} & -2 c_{6} & 2 c_{2}-2 c_{3} \\
c_{8} & c_{4} & 2 c_{2}-2 c_{3} & 2 c_{6}
\end{array}\right) .
$$

The line (17) is contained in it if and only if $\mathbf{p}^{\top} C_{2} \mathbf{q}=0$. This condition is equivalent to

$$
\begin{array}{r}
0=\left(-c_{5} c_{8}^{2}+c_{4} c_{8} c_{9}\right) t+\left(c_{4} c_{9}^{2}-c_{5} c_{8} c_{9}\right) s \\
+2\left(c_{2}-c_{3}\right) c_{9} c_{8}+\left(c_{9}^{2}-c_{8}^{2}\right) c_{6},
\end{array}
$$

which is bi-linear in $(s, t)$. We can solve it directly for $t=$ $t(s)$. Now, by intersecting the line $\tilde{\mathbf{r}}(s, t(s), u)$ with the other two quadrics $Q_{1}$ and $Q_{3}$ we arrive at two quadratic equations which can be solved for $u=u(s)$. After eliminating the trivial solutions $\mathbf{p}(s)$ and $\mathbf{q}(t(s))$, respectively, we obtain for each of these two quadratic equations the same solution $u(s)$. Thus, we have found a parameterization of the common cubic intersection curve $\mathbf{r}(s, t(s), u(s))$ of the three quadric surfaces.

Now we formulate the main result of this section.

Theorem 1: Two quadratically supported surfaces possess at most six common normals.

Proof: Two QSS possess a common normal if and only if the equations (11) and (12) hold. According to lemma 2, the intersection of the three quadrics of Eq. 11 is a cubic curve. Consequently, by intersecting it with the unit sphere (i.e., equation (12)) we get at most six common normals.

The proofs suggest the following algorithm for computing the joint normals of two QSS:

1) Generate a cubic rational parameterization of the joint intersection curve of the three quadric surfaces (11), according to the proof of Lemma 2.

2) Compose the parameterization with (12) in order to find a degree 6 polynomial.

3) Use an appropriate numerical method to find all roots of this polynomial.

4) Evaluate the parameterization of the cubic at these roots and apply the envelope operators (8) to the resulting unit normals.

\section{EXAMPLES}

As a first example we consider two convex QSS (see Figure 5), denoted by $C_{1}$ (blue) and $C_{2}$ (orange). They are defined by their coefficients $a$ and $b$ according to section III

$$
C_{1}: a=\frac{2}{3}, b=1, C_{2}: a=\frac{5}{7}, b=\frac{6}{7}
$$

and some additional translation, rotation and scaling. Computing the minimum distance via our presented algorithm above leads us to the polynomial equation

$$
\begin{aligned}
0=x^{6}-\frac{7983}{886} x^{5} & +\frac{48439}{1772} x^{4}-\frac{12933}{433} x^{3}+ \\
& +\frac{425}{886} x^{2}+\frac{23877}{1772} x-\frac{12635}{3544},
\end{aligned}
$$

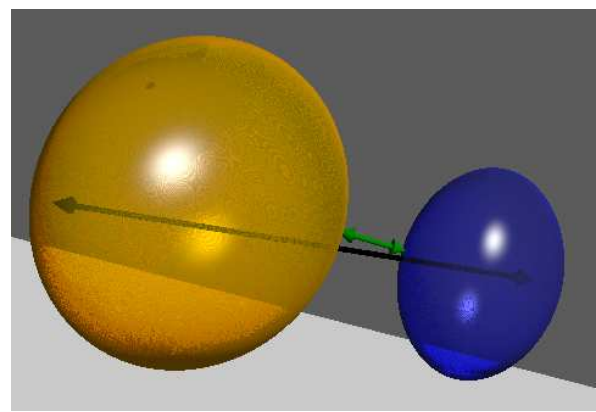

Fig. 5. Shortest (green) and longest distance (black) between two convex objects represented by QSS.

which has been solved numerically. It yields exactly two real roots, that correspond to the longest and the shortest distance of the two convex QSS.

In the second example we compute the shortest distances between a moving manipulator-type object and a convex obstacle (see Figure 1). The manipulator has been modeled as a collection of two non-convex QSS of revolution and three spheres (also described by QSS). Due to the geometry of these surfaces, the shortest distances are always realized at normals intersecting the surfaces at regular points, hence it is not necessary to check the singularities (which are located along circles) of the non-convex QSS separately. The obstacle is modeled as a convex QSS.

The manipulator performs a motion, and we computed the shortest distance to the obstacle for several positions of the motion, see Figure 1. For each position, the roots of 5 polynomials of degree 6 each had to be found, and the common normal corresponding to the shortest distance was then identified.

\section{THE PLANAR CASE}

Finally we discuss the case of planar objects with quadratic polynomial support functions. In the planar case any quadratic support function has the normal form $h(\mathbf{n})=$ $x^{2}+a y^{2}$ with $a \in \mathbb{R}$. We call the corresponding envelopes quadratically supported curves (QSC). They possess the trigonometric parameterization

$$
\mathbf{x}_{h}(\cos \phi, \sin \phi)=\left(\begin{array}{c}
\left(2-a+(a-1) \cos ^{2} \phi\right) \cos \phi \\
\left(a+(a-1) \cos ^{2} \phi\right) \sin \phi
\end{array}\right) .
$$

The possible shapes of the QSC depend on the parameter $a$ and can be found in the Figure 6 .

Let us consider two quadratic support functions

$$
\begin{aligned}
& g(\mathbf{n})=a_{1} x^{2}+a_{2} y^{2}+a_{3} x y+a_{4} x+a_{5} y+a_{6} \text { and } \\
& h(\mathbf{n})=b_{1} x^{2}+b_{2} y^{2}+b_{3} x y+b_{4} x+b_{5} y+b_{6},
\end{aligned}
$$

with constant coefficients $a_{i}, b_{i} \in \mathbb{R}$ for $i=1 \ldots 6$. By using the same arguments introduced in Section IV, both QSC posses a common normal if and only if $\nabla g(\mathbf{n})-\nabla h(\mathbf{n})$ and $\mathbf{n}$ are linearly dependent. This leads to the following non-linear system of equations

$$
\begin{aligned}
& 0=c_{3}\left(x^{2}-y^{2}\right)-2\left(c_{1}-c_{2}\right) x y+c_{5} x-c_{4} y \\
& 1=x^{2}+y^{2}
\end{aligned}
$$



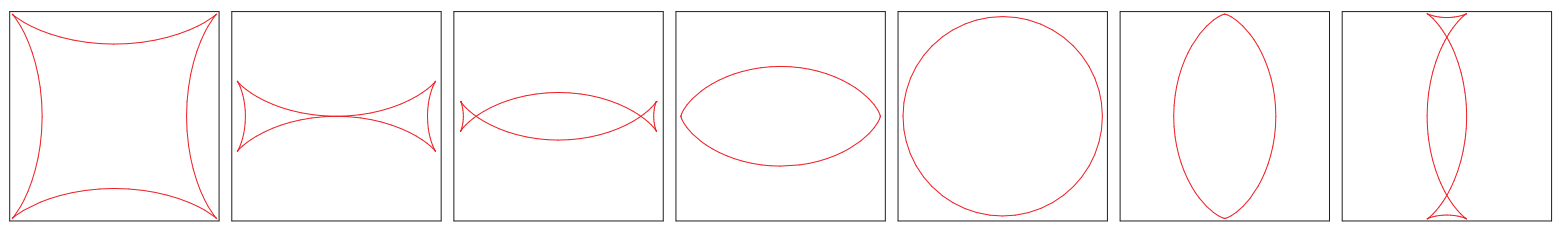

Fig. 6. Shapes of a QSC: $a \in(-\infty, 0), a=0, a \in\left(0, \frac{1}{2}\right), a=\frac{1}{2}, a \in\left(\frac{1}{2}, 2\right), a=2, a \in(2,+\infty)$ (from left to right).

where $c_{i}=a_{i}-b_{i}$ for $i=1 \ldots 5$. The change of variables

$$
x=\frac{1-u^{2}}{1+u^{2}}, \quad y=\frac{2 u}{1+u^{2}}
$$

produces the quartic equation in $u$

$$
\begin{gathered}
0=\left(c_{3}-c_{5}\right) u^{4}+2\left(2 c_{1}-2 c_{2}-c_{4}\right) u^{3}-6 c_{3} u^{2} \\
-2\left(2 c_{1}+c_{4}-2 c_{2}\right) u+c_{3}+c_{5}
\end{gathered}
$$

whose real solutions provide the common normals between the considered QSC after applying the envelope operators (8) to the resulting unit normals.

\section{CONCLUSION}

We presented a new and promising geometric primitive which can be used as a bounding volume for applications related to collision detection and shortest distance computation. It has the same number of degrees of freedom as a general quadric surface (9). However, while the computation of the shortest distance of two quadric surfaces leads to a degree 24 polynomial, and examples with 16 common real normals have been reported [21], the same computation for our new bounding primitive requires only to solve a polynomial of degree 6.

Based on these theoretical results, we have implemented an algorithm for minimum distance computation between free form objects, which are represented as a union of QSS. The results are described in the forthcoming paper [15]. As shown there, the shortest distance computation between pairs of QSS requires roughly $30 \mu s$ on standard hardware. This compares well with analogous results for pairs of ellipsoids, which were reported in [2].

\section{REFERENCES}

[1] Chen, X.-D., Yong, J.-H., Zheng, G.-Q, Paul, J.-C. and Sun J.-G. (2006), Computing minimum distance between two implicit algebraic surfaces, Computer-Aided Design, vol. 38, no. 10, pp. 1053-1061.

[2] Choi, Y.-K., Wang, W. and Kim, M.-S. (2003), Exact Collision Detection of Two Moving Ellipsoids under Rational Motions, Proceedings of the 2003 International Conference on Robotics \& Automation, IEEE Press, Taipei, pp. 349-354.

[3] Eberly, D. H. (2001). 3D Game Engine Design, Academic Press.

[4] Ericson, C. (2004). Real-Time Collision Detection, Morgan Kaufmann.

[5] Gravesen J. (2007), Surfaces parametrised by the normals, Computing, vol. 79, pp. 175-183.

[6] Gruber, P., Wills, J. (Eds.) (1993). Handbook of Convex Geometry. North-Holland, Amsterdam.

[7] Ju, M., Liu, J., Shiang, S., Chien, Y., Hwang, K. and Lee, W. (2001). A novel collision detection method based on enclosed ellipsoid, Proceedings of the 2001 IEEE Conference on Robotics \& Automation, pp. 21-26.

[8] Kim K.-J. (2003), Minimum distance between a canal surface and a simple surface, Computer-Aided Design, vol. 35, no. 10, pp. 871-879.
[9] Klosowski, J., Held, M., Mitchell, J.S.B., Sowizral, H. and Zikan, H. (1998), Efficient collision detection using bounding volume hierarchies of k-DOPs, IEEE Transactions on Visualization and Computer Graphics, vol. 4, pp. 21-36.

[10] Lennerz, C. and Schömer, E. (2002), Efficient Distance Computation for Quadratic Curves and Surfaces, GMP '02: Proceedings of the Geometric Modeling and Processing - Theory and Applications (GMP'02), IEEE Press, 60-69.

[11] Lee, K., Seong, J.-K., Kim, K.-J. and Hong, S.J. (2007), Minimum distance between two sphere-swept surfaces, Computer Aided Design, vol. 39 , no. 6 , pp. $452-459$.

[12] Oberneder, M., Jüttler, B. and Gonzalez-Vega, L. (2008), Exact envelope computation for moving surfaces with quadratic support functions, J. Lenarčič, P. Wenger, Advances in Robot Kinematics Analysis and Design, Springer, pp. 283-290.

[13] Odehnal, B. (2005), Common Normals of Two Tori, Journal for Geometry and Graphics, vol. 9, pp. 51-65.

[14] Perram, J., Rasmussen, J., Prastaard, E. and Lebowitz, J. (1996), Ellipsoids contact potential: theory and relation to overlap potentials, Physics Review E, vol. 54, no. 6, pp. 6565-6572.

[15] Rabl M., Jüttler B. (2009), Fast Distance Computation Using Quadratically Supported Surfaces. FSP report no. 80, available at http: / /www. industrial-geometry.at/techrep.php

[16] Rimon, E. and Boyd, S.P. (1997), Obstacle collision detection using best ellipsoid fit, Journal of Intelligent and Robotic Systems, vol. 18, pp. $105-126$.

[17] Sabin, M., (1974). A Class of Surfaces Closed under Five Important Geometric Operations. Technical Report VTO/MS/207, British Aircraft Corporation, available at http://www.damtp.cam. ac.uk/user/na/people/Malcolm/vtoms/vtos.html.

[18] Shiang, S.P., Liu, J.S. and Chien, Y.R. (2000) Estimate of minimum distance between convex polyhedra based on enclosed ellipsoids, Proceedings of the IEEE/RST International Conference on Intelligent Robots and Systems, Takamatsu, Japan, pp. 739-744.

[19] Sir, Z., Gravesen, J., and Jüttler, B. (2008), Curves and surfaces represented by polynomial support functions, Theoretical Computer Science, vol. 392, pp. 141-157.

[20] Seong, J.-K., Kim M.-S. and Sugihara, K. (2002), The Minkowski Sum of Two Simple Surfaces Generated by Slope-Monotone Closed Curves, GMP '02: Proceedings of the Geometric Modeling and Processing Theory and Applications (GMP'02), Washington, DC, USA.

[21] Sohn, K.-A., Jüttler, B., Kim, M.-S. and Wang, W. (2002), Computing the distance between two surfaces via line geometry, Proceedings of the tenth Pacific conference on computer graphics and applications, IEEE Press, Los Alamitos, pp. 236-245.

[22] Wang, W. (2002), Modeling and Processing with Quadric Surfaces, G. Farin, J. Hoschek, and M.-S. Kim, (Eds.), Handbook of Computer Aided Geometric Design, North-Holland, pp. 777-795.

[23] Wang, W., Goldman, R. and Tu,C. (2003) Enhancing Levin's method for computing quadric-surface intersections. Computer Aided Geometric Design, vol. 20, no. 7, pp. 401-422.

[24] Wang, W., Wang, J. and Kim, M.-S. (2001) An algebraic condition for the separation of two ellipsoids, Computer Aided Geometric Design, vol. 18 , no. 6 , pp. 531-539.

[25] Wu, C.J. (1996) On the representation and collision detection of robots, Journal of Intelligent and Robotic Systems, vol. 16, pp. 151-168. 